\title{
Quantifying cerebral hypoxia by near-infrared spectroscopy tissue oximetry: the role of arterial-to- venous blood volume ratio
}

Martin B. Rasmussen

Vibeke R. Eriksen

Bjørn Andresen

Simon Hyttel-Sørensen

Gorm Greisen 


\title{
Quantifying cerebral hypoxia by near-infrared spectroscopy tissue oximetry: the role of arterial-to-venous blood volume ratio
}

\author{
Martin B. Rasmussen, ${ }^{\mathrm{a}, \mathrm{b}, *}$ Vibeke R. Eriksen, ${ }^{\mathrm{a}, \mathrm{b}}$ Bjørn Andresen, ${ }^{\mathrm{a}}$ Simon Hyttel-Sørensen, ${ }^{\mathrm{a}}$ and \\ Gorm Greisen ${ }^{\mathrm{a}}$ \\ ${ }^{a}$ Copenhagen University Hospital—Rigshospitalet, Department of Neonatology, Blegdamsvej 9, Copenhagen 2100, Denmark \\ bUniversity of Copenhagen, Faculty of Health and Medical Sciences, Blegdamsvej 3, Copenhagen 2200, Denmark
}

\begin{abstract}
Tissue oxygenation estimated by near-infrared spectroscopy (NIRS) is a volume-weighted mean of the arterial and venous hemoglobin oxygenation. In vivo validation assumes a fixed arterial-tovenous volume-ratio (AV-ratio). Regulatory cerebro-vascular mechanisms may change the AV-ratio. We used hypotension to investigate the influence of blood volume distribution on cerebral NIRS in a newborn piglet model. Hypotension was induced gradually by inflating a balloon-catheter in the inferior vena cava and the regional tissue oxygenation from $\mathrm{NIRS}\left(\mathrm{rStO}_{2, \mathrm{NIRS}}\right)$ was then compared to a reference $\left(\mathrm{rStO}_{2, \mathrm{COx}}\right)$ calculated from superior sagittal sinus and aortic blood sample co-oximetry with a fixed AV-ratio. Apparent changes in the AV-ratio and cerebral blood volume (CBV) were also calculated. The mean arterial blood pressure (MABP) range was 14 to $82 \mathrm{mmHg} . \mathrm{PaCO}_{2}$ and $\mathrm{SaO}_{2}$ were stable during measurements. $\mathrm{rStO}_{2, \mathrm{NIRS}}$ mirrored only $25 \%(95 \% \mathrm{Cl}: 21 \%$ to $28 \%, p<0.001)$ of changes in $\mathrm{rStO}_{2, \mathrm{cox}}$. Calculated AVratio increased with decreasing MABP (slope: $-0.007 \cdot \mathrm{mmHg}^{-1}, p<0.001$ ). NIRS estimates that CBV decreased with decreasing MABP (slope: $0.008 \mathrm{ml} / 100 \mathrm{~g} / \mathrm{mmHg}, p<0.001$ ). Thus, cerebral NIRS oximetry responded poorly to changes in tissue oxygenation during hypotension induced by decreased preload. An increase in the AV-ratio during hypotension due to arterial vasodilation and, possibly, cerebral venous collapse may be a part of the explanation. ( The Authors. Published by SPIE under a Creative Commons Attribution 3.0 Unported License. Distribution or reproduction of this work in whole or in part requires full attribution of the original publication, including its DOI. [DOI: 10.1117/1. JBO.22.2.025001]
\end{abstract}

Keywords: cerebral near-infrared spectroscopy; arterial-to-venous volume-ratio; hypotension; experimental design; neonatal. Paper 160680R received Oct. 1, 2016; accepted for publication Jan. 6, 2017; published online Feb. 2, 2017.

\section{Introduction}

The immature cardiovascular system of preterm infants puts them at risk for low blood pressure (hypotension); a condition known to be associated with cerebral injury. ${ }^{1-3}$ However, the lower threshold of blood pressure leading to reduced brain perfusion has not yet been established in preterm infants ${ }^{4,5}$ and it might very well vary both within and between infants. Consequently, more direct methods of monitoring the sufficiency of oxygen supply to the brain are under investigation. Near-infrared spectroscopy (NIRS) is a noninvasive method that allows for measurement of cerebral oxygenation. ${ }^{6}$ Continuous monitoring with NIRS to guide treatment has been demonstrated to reduce the hypoxic burden on the brain in extremely preterm infants. ${ }^{7}$ The optical properties of hemoglobin change with the binding of oxygen. NIRS illuminates tissue with near-infrared light and detects the reflected light at some distance from the light source. ${ }^{8}$ Since hemoglobin in all vascular compartments within the illuminated tissue contributes to the attenuation of the reflected light, a reference for in vivo validation from co-oximetry, i.e., direct blood gas analysis from blood samples, must be a weighted average reflecting the distribution between venous and arterial volume. A cerebral

*Address all correspondence to: Martin B. Rasmussen, E-mail: martin.bo. rasmussen10@gmail.com
What we already know about this topic

- NIRS tissue oximetry enables noninvasive estimation of the hemoglobin oxygen saturation in a regional vascular bed.

- In vivo validation of NIRS tissue oximetry depends on a fixed arterial-to-venous blood volume ratio for the calculation of a reference value from co-oximetry on arterial and regional venous blood.

- Cerebral arterial-to-venous blood volume ratio changes with hypoxia and hypercapnia, due to regulatory vasoreactivity.

What this study adds that is new

- When arterial hypotension was induced by obstruction of blood flow in the inferior vena cava, the drop in cerebral oxygenation as measured by NIRS was significantly less than the drop measured by co-oximetry.

- This relative insensitivity of cerebral oximetry to ischemic hypoxia may at least partly be due to an increase in the AV ratio during hypotension. 
arterial-to-venous volume ratio (AV-ratio) of 1 to $3(0.25)$ is widely accepted in humans under normal physiological conditions. ${ }^{6,9}$ However, the cerebral blood volume (CBV) distribution might change over time due to cerebral autoregulation; the ability of blood vessels to regulate arterial resistance to maintain a stable perfusion despite blood pressure changes, through chemical, metabolic, and neurogenic mechanisms. ${ }^{10}$ Accordingly, pathophysiological conditions known to effect cerebral perfusion, such as low tissue oxygenation (hypoxia), elevated blood levels of carbon dioxide (hypercapnia), or hypotension (low arterial blood pressure) may change the AV-ratio. Previously, NIRS oximetry validation against arterial and cerebral venous blood oxygenation measured by co-oximetry during hypoxia has suggested changes in the AV-ratio. ${ }^{11}$ The change in AV-ratio estimated by NIRS during hypotension has not yet been examined. Improving our knowledge about changes in the cerebral AV-ratio during pathologic states could strengthen the interpretation of data obtained by NIRS, as well as improve the accuracy when validated against cerebral blood co-oximetry.

\section{Methods}

\subsection{Animals Included}

We used a dataset obtained during a previous study on the effects of dopamine on the cerebral autoregulation in hypotensive newborn piglets. We used the dataset to compare the values of cerebral oxygenation obtained by NIRS and co-oximetry. Accordingly, the piglets had continuous dopamine infusion during half of the experiment, as described in Sec. 2.5.

\subsection{Anesthesia and Monitoring}

Anesthesia was induced by $3 \%$ isoflourane, supplemented with $5 \mathrm{mg} / \mathrm{kg}$ propofol and $20 \mu \mathrm{g} / \mathrm{kg}$ fentanyl. Induction of anesthesia was followed by continuous infusion of propofol 15 to $20 \mathrm{mg} / \mathrm{kg} / \mathrm{h}$ and fentanyl $7 \mu \mathrm{g} / \mathrm{kg} / \mathrm{h}$. The fentanyl infusion was stopped $1 \mathrm{~h}$ prior to the start of planned blood pressure manipulations, as described in Sec. 2.5. Piglets were tracheostomized and connected to a pressure-controlled mechanical ventilator. $\mathrm{PaCO}_{2}$ was maintained between 4 to $6 \mathrm{kPa}$ and arterial saturation above $95 \%$. Arterial saturation and heart rate were monitored by pulse oximetry on the foreleg (Radical 7, Masimo, Irvine, California). Piglets were kept on a heating pad to maintain rectal temperature at $38.5^{\circ} \mathrm{C}$ to $39.5^{\circ} \mathrm{C}$.

\subsection{Surgical Preparation}

The femoral artery was cannulated with an arterial line placed in the thoracic aorta for mean arterial blood pressure (MABP) monitoring and arterial blood sampling. The femoral veins were cannulated with a 4 FR double lumen catheter for infusion of glucose and dopamine and a 4 FR embolectomy catheter with a balloon at its tip (LeMaitre Vascular, Sulzbach, Germany). The balloon was placed in the inferior vena cava (16 cm mark) to decrease cardiac preload and MABP when inflated. Control of placement of the embolectomy catheter was done by making sure that MABP was easily manipulated during inflation/deflation. Cerebral venous blood samples were retrieved by cannulating the superior sagittal sinus. The piglets recovered for $1 \mathrm{~h}$ after preparation and cannulation.

\subsection{Cerebral Monitoring}

We used the NIRO 300 (Hamamatsu Phototonics, Hamamatsu City, Japan) with an NIRS probe consisting of two aligned photodetectors and a light-source, which emits near-infrared light at four different wavelengths. The photodetectors were placed $4 \mathrm{~cm}$ from the emission probe in a nontransparent probe holder. The NIRS probe was placed above the left frontal and parietal cortex. The position of the NIRS probe was secured with a selfadhesive bandage and, once placed, the NIRS probe was not relocated. The device estimates change in oxy-hemoglobin and deoxy-hemoglobin through spatially resolved spectroscopy by measuring light attenuation at the two source-detector separations. ${ }^{8}$ Regional tissue oxygenation $\left(\mathrm{rStO}_{2, \mathrm{NIRS}}\right)$, i.e., the regional oxygenated hemoglobin to total hemoglobin in percentage was estimated at $2 \mathrm{~Hz}$. $\mathrm{rStO}_{2, \mathrm{NIRS}}$ is labeled "tissue oxygenation index" by the manufacturer; Hamamatsu Phototonics.

\subsection{Experimental Design}

MABP was manipulated by inflating or deflating the balloon in the inferior vena cava. Changes in blood pressure were induced gradually in steps of $\sim 10 \mathrm{mmHg}$. At each stable MABP level, gas analysis (ABL-500, Radiometer, Copenhagen, Denmark) was done on blood drawn from the arterial line and superior sagittal sinus. The sampled blood volume was replaced with saline. Piglets were randomized to start with either baseline, i.e., blood pressure with a completely deflated balloon, or with low blood pressure. Hence, all piglets had a phase of gradual increase and a phase of gradual decrease of MABP, but the order of the phases was randomized. If the piglet was randomized to start with baseline blood pressure, MABP was lowered to a minimum of $\sim 20 \mathrm{mmHg}$. Subsequently, MABP was returned to baseline blood pressure. If piglets were randomized to start with low blood pressure, MABP was gradually returned to baseline blood pressure and, subsequently, gradually lowered to a minimum of $\sim 20 \mathrm{mmHg}$. Between the two phases of either increase or decrease in MABP, the piglet was allowed to stabilize with the balloon fully deflated for $30 \mathrm{~min}$.

As part of a larger experimental design, the piglets were randomized to receive continuous dopamine infusion during either the phase of increase or decrease in MABP.

\subsection{Changes in Cerebral Blood Volume}

Changes in $\mathrm{CBV}$ from baseline $(\triangle \mathrm{CBV})$ were calculated by dividing changes in hemoglobin from baseline measured by NIRS $(\triangle \mathrm{HBT})$ with total hemoglobin measured with arterial co-oximetry (HBT). ${ }^{12}$ NIRS measurements were converted to $\mu \mathrm{mol} / \mathrm{l}$ by using a differential path length factor (DPF) of 4.99. ${ }^{13} \triangle \mathrm{CBV}$ in $\mathrm{ml}$ per $100 \mathrm{~g}$ was calculated according to the following equation: ${ }^{14}$

$$
\Delta \mathrm{CBV}=\frac{\Delta \mathrm{HBT} * \mathrm{MW}_{\text {hemoglobin }} * 10^{-6}}{\mathrm{HBT} * 10^{-2} * \mathrm{CLVHR} * D t * 10},
$$

where $\mathrm{MW}_{\text {hemoglobin }}$ is the molecular weight of hemoglobin = 64,500 D, CLVHR is cerebral to large vessel haematocrit ratio $=0,69,{ }^{15}$ and $D t$ is the brain tissue density = $1.05 \mathrm{~g} \cdot \mathrm{ml}^{-1} .16$ 


\subsection{Data Analysis}

We used the mean of $15 \mathrm{~s}$ of $\mathrm{rStO}_{2, \mathrm{NIRS}}$ measurements after arterial and venous sampling. Assuming an AV-ratio of 0.25 , the reference $\mathrm{rStO}_{2, \mathrm{COX}}$ was calculated by the following equation:

$\mathrm{rStO}_{2, \mathrm{COX}}=\frac{1}{4} * \mathrm{~S}_{\mathrm{a}} \mathrm{O}_{2}+\frac{3}{4} * \mathrm{~S}_{\mathrm{v}} \mathrm{O}_{2}$.

Assuming $\mathrm{rStO}_{2, \mathrm{NIRS}}$ to be the true regional cerebral oxygenation and that all blood exists in either the arterial or venous compartment, it is possible to calculate arterial and venous volume fractions by solving the following equation for $A$ and $V:^{11}$

$$
\begin{aligned}
\mathrm{rStO}_{2, \mathrm{NIRS}} & =A * \mathrm{~S}_{\mathrm{a}} \mathrm{O}_{2}+V * \mathrm{~S}_{\mathrm{v}} \mathrm{O}_{2}, A+V=1, \\
\text { Solved to: } A & =\frac{\mathrm{rStO}_{2, \mathrm{NIRS}}-\mathrm{S}_{\mathrm{v}} \mathrm{O}_{2}}{\mathrm{~S}_{\mathrm{a}} \mathrm{O}_{2}-\mathrm{S}_{\mathrm{v}} \mathrm{O}_{2}},
\end{aligned}
$$

where $A$ is the arterial blood volume fraction and $V$ is the venous volume fraction. This allowed the AV-ratio to be calculated at different levels of MABP. Changes in MABP, $\mathrm{rStO}_{2, \mathrm{NIRS}}$, and $\mathrm{rStO}_{2, \mathrm{COX}}$ were described with median, range, and interquartile range (IQR). Using regression analysis, we investigated the relationship between $\mathrm{rStO}_{2, \mathrm{NIRS}}$ and $\mathrm{rStO}_{2, \mathrm{COX}}$. Similarly, regression analysis was used to describe the relationship between changes in MABP from baseline and estimations of $\mathrm{AV}$-ratio and $\triangle \mathrm{CBV}$, respectively. In order to account for repeated measurements within piglets, linear random effect models were used to describe all relationships. Results not physiologically possible, such as the AV-ratio above 1 and below 0 , were included throughout the analysis to avoid statistical bias. It follows that $A<0$ can only be achieved if $\mathrm{S}_{v} \mathrm{O}_{2}>\mathrm{rStO}_{2, \mathrm{NIRS}}$.

\subsection{Ethics}

The Danish Animal Experiments Inspectorate approved the experimental protocol: 2014-15-0201-00123. At the end of the experiment, piglets were euthanized with pentobarbitone (100 mg/kg) while still anesthetized.

\section{Results}

\subsection{Piglets and Descriptive Statistics}

Twenty-one piglets were studied with a median age of $60 \mathrm{~h}$ (range 4 to 66) and mean weight $1.9 \pm 0.2 \mathrm{~kg}$. A total of 246 paired measurements were analyzed. The number of data pairs in each piglet ranged from 10 to 13 . A total of five pairs of measurements were excluded; one due to erroneous NIRS-measurement, and four due to failed blood gas analysis. For all piglets, $\mathrm{S}_{\mathrm{a}} \mathrm{O}_{2}$ was kept above $95 \%$. $\mathrm{pCO}_{2}$ was stable at $5.1 \pm 0.8 \mathrm{kPa}$ during the phases with changing MABP.

\section{2 $\mathrm{rStO}_{2, \mathrm{NIRS}}-\mathrm{rStO}_{2, \mathrm{COx}}$ Relationship, AV-Ratio, and $\triangle \mathrm{CBV}$}

An example of concurrent changes in MABP, $\mathrm{rStO}_{2, \mathrm{NIRS}}$, and $\mathrm{rStO}_{2, \mathrm{COX}}$ during the experiment is given in Fig. 1. The average change in $\mathrm{rStO}_{2, \mathrm{NIRS}}$ was $25 \%$ of the corresponding change in $\mathrm{rStO}_{2, \mathrm{COX}}$ (Fig. 2). CBV decreased with $0.008 \pm 0.001 \mathrm{ml}$ per $100 \mathrm{~g}$ per mmHg decrease in MABP (Fig. 3). Median AV-ration

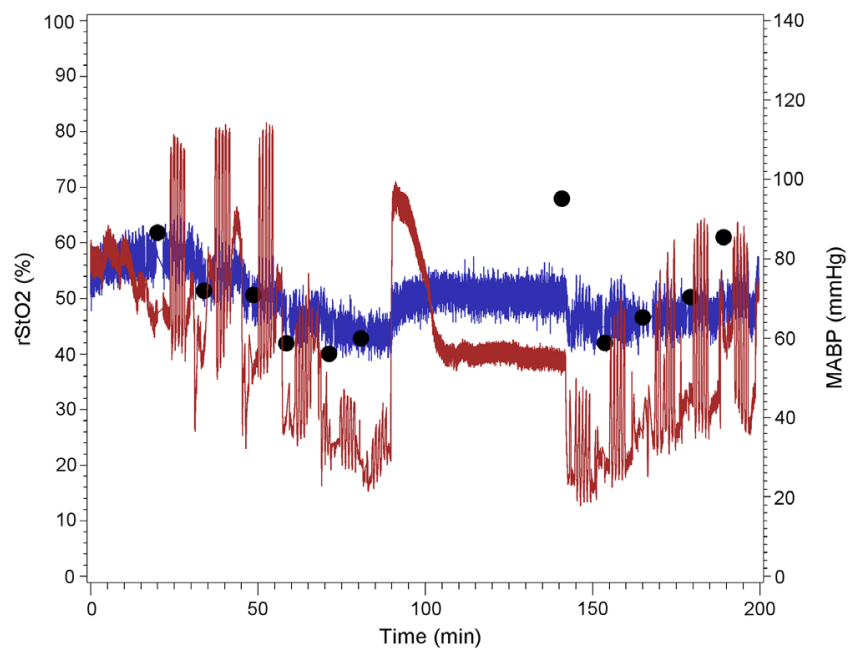

Fig. 1 An example of changes in cerebral oxygenation $\left(\mathrm{rStO}_{2}\right)$ measured by NIRS ( $\mathrm{rStO}_{2, \mathrm{NIRS}}$ ), blue line, reference tissue oxygenation $\left(\mathrm{rStO}_{2, \mathrm{COx}}\right)$, black dots, and changes in MABP, red line, throughout the experiment. MABP was decreased stepwise by inflating a balloon in the inferior vena cava. At each level of MABP, blood gas analysis was done to calculate $\mathrm{rStO}_{2, \mathrm{Cox}}$. As part of a parallel experiment, MABP was alternately increased and decreased for 5 min with an arterial balloon placed in thoracic aorta at each MABP level. Blood sampling was done prior to this and arterial and venous oxygenations were not affected by the MABP fluctuations. The piglets were allowed to rest for $\sim 30 \mathrm{~min}$ between the first and the second half of the experiment. In the second half, MABP was gradually increased by deflating the vena cava inferior balloon. Piglets were randomized to start with baseline MABP or low MABP and, as part of the parallel experiment, to have dopamine infusion during either the first or the second half of the experiment. The shown example started with baseline MABP and dopamine during the first half of the experiment.

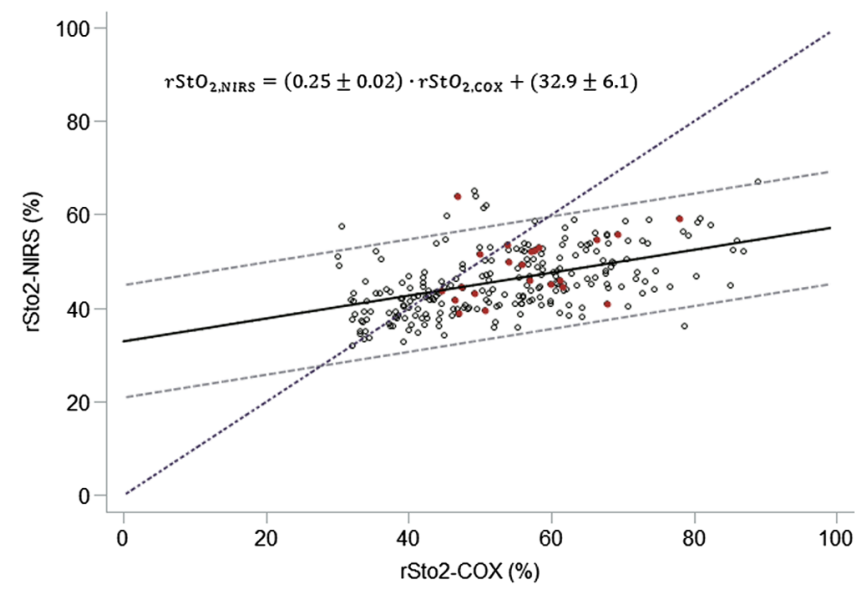

Fig. 2 Cerebral oxygenation in percent measured by NIRS $\left(\mathrm{rStO}_{2}-\mathrm{NIRS}\right)$ plotted against a reference tissue oxygenation $\left(\mathrm{rStO}_{2}-\mathrm{COX}\right)$ calculated as a weighted average of oxygenation in arterial and sagittal sinus blood in a fixed ratio of 0.25 . Brown dots are baseline values. Black circles are measurements during hypotension. The average changes in $\mathrm{rStO}_{2}-\mathrm{NIRS}$ were $25 \%(p<0.001)$ of the corresponding changes in $\mathrm{rStO}_{2}-\mathrm{COX}$. The purple, dotted line indicates line of equity.

at baseline was 0.17 . The AV-ratio was inversely correlated to MABP increasing by $0.007 \mathrm{mmHg}^{-1}$ (Fig. 4). Estimates of the $\mathrm{AV}$-ratio were above 1 or below 0 in 81 out of 246 data points with 79 being below 0 . 


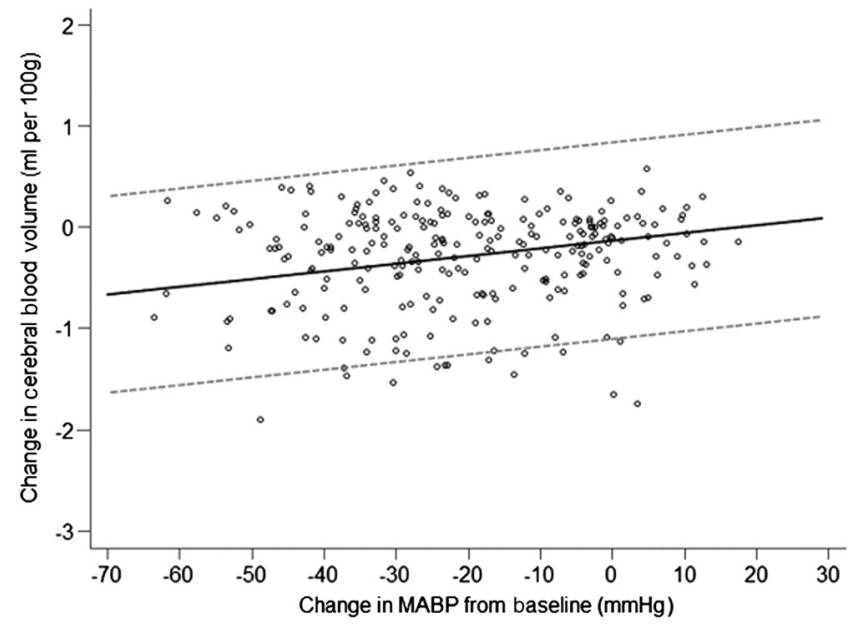

Fig. 3 Change in CBV in $\mathrm{ml} \mathrm{pr.} 100 \mathrm{~g}$ from the start of the experiment against change in mean arterial pressure (MABP).

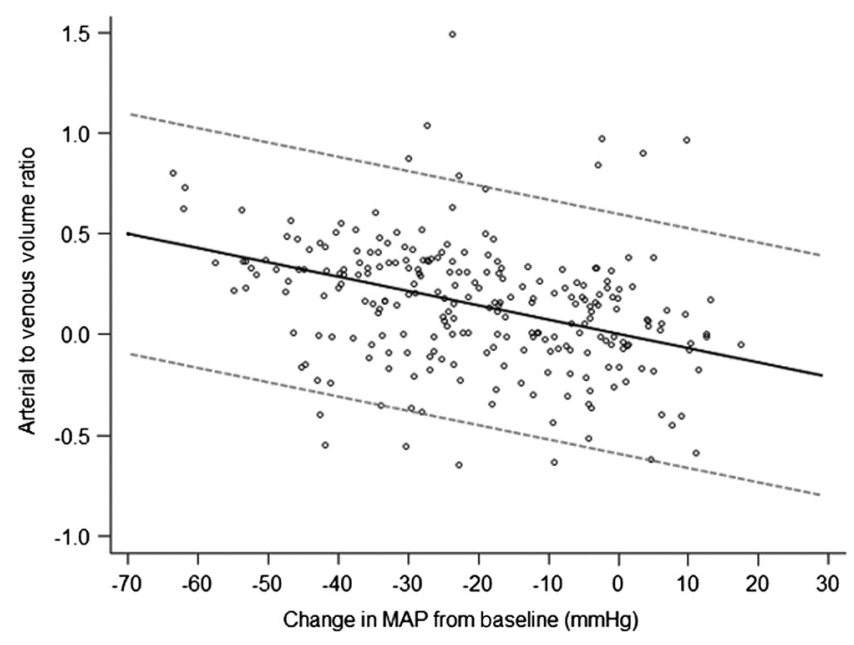

Fig. 4 Calculation of fractional contribution of arterial and venous blood volume to NIRS estimates from $\mathrm{S}_{\mathrm{a}} \mathrm{O}_{2}, \mathrm{~S}_{\mathrm{v}} \mathrm{O}_{2}$, and $\mathrm{rStO}_{2, \mathrm{NIRS}}$, assuming that all NIRS hemoglobin signal come from arterial or venous vascular beds. Arterio-to-venous volume fraction increases by 0.007 with each $\mathrm{mmHg}$ drop in MABP. (Values of the AV-ratio outside the interval 0 to 1 are impossible, but all data were retained to avoid bias in the statistical analysis).

\subsection{Randomization}

None of the outcome variables; $\mathrm{rStO}_{2, \mathrm{NIRS}}$, the $\mathrm{AV}$-ratio, or $\triangle \mathrm{CBV}$, were affected by whether or not the piglet started with low or baseline MABP. Similarly, it made no difference if dopamine was infused in the first or second half of the experiment (Table 1).

\section{Discussion}

This is, to our knowledge, the first study to investigate the influence of hypotension on apparent blood volume redistribution with cerebral NIRS oximetry. During a wide range of blood pressures, our data showed that NIRS oximetry poorly reflect changes in reference cerebral oximetry. The apparent AVratio increased as the MABP was reduced. This may in part
Table 1 Descriptive statistics and regression statistics.

\begin{tabular}{|c|c|c|}
\hline Parameter & Baseline & Lowest MABP \\
\hline MABP $(\mathrm{mmHg})$ & 61.6 (57.9 to 69.7$)$ & 21.1 (18.9 to 21.5$)$ \\
\hline $\mathrm{rStO}_{2, \mathrm{NIRS}}(\%)$ & 47.8 (43.8 to 53.2 ) & 39.9 (37.3 to 42.1$)$ \\
\hline $\mathrm{rStO}_{2, \operatorname{cox}}(\%)$ & 56.4 (49.2 to 61.2$)$ & 38.9 (34.1 to 44.6$)$ \\
\hline Regression & Slope & $p$ value \\
\hline $\begin{array}{l}\mathrm{rStO}_{2, \mathrm{NIRS}} \\
\text { versus } \mathrm{rStO}_{2, \mathrm{COx}} \text { (fraction) }\end{array}$ & $0.25 \pm 0.02$ & $p<0.001$ \\
\hline $\begin{array}{l}\text { CBV versus MABP } \\
\left(\mathrm{ml} \cdot 100 \mathrm{~g}^{-1} \cdot \mathrm{mmHg}^{-1}\right)\end{array}$ & $0.008 \pm 0.001$ & $p<0.001$ \\
\hline $\begin{array}{l}\text { AV-ratio versus } \\
\text { MABP }\left(\mathrm{mmHg}^{-1}\right)\end{array}$ & $0.007 \pm 0.002$ & $p<0.001$ \\
\hline
\end{tabular}

Note: MABP, $\mathrm{rStO}_{2, \mathrm{NIRS}}$, and $\mathrm{rStO}_{2, \mathrm{cox}}$ data described with median (IQR). Regression slopes \pm SEM during hypotension. The slope is different from 0 if $p<0.05$. MABP, mean arterial blood pressure.

explain the inaccuracy of the cerebral NIRS estimates of $\mathrm{rStO}_{2, \mathrm{COX}}$.

\subsection{Reactivity of Cerebral NIRS Oximetry Assuming a Fixed AV-Ratio}

A volume-weighted mean of the arterial and venous oxygenation is used for in vivo calibration of cerebral NIRS oximetry. ${ }^{17}$ Pollard et al. ${ }^{6}$ showed that cerebral NIRS oximetry reflected cerebral oxygenation in an AV-ratio of 0.25 in healthy adult volunteers. This fixed AV-ratio is widely accepted in literature, although some studies suggest wide interindividual variation. ${ }^{18,19}$ Assuming this fixed AV-ratio of 0.25 to calculate a reference value of tissue saturation from co-oximetry, we found that $\mathrm{rStO}_{2, \mathrm{NIRS}}$ changed considerably less than expected: At low cerebral oxygenation, $\mathrm{rStO}_{2, \mathrm{NIRS}}$ measured higher than $\mathrm{rStO}_{2, \mathrm{COX}}$, and at high oxygenation $\mathrm{rStO}_{2, \mathrm{NIRS}}$ measurements were lower than $\mathrm{rStO}_{2, \mathrm{COX}}$. The NIRO-300 used in this study has previously been seen to react less to changes in oxygenation than the INVOS 5100 instrument (INVOS, Covidien, Boulder), which utilize the same spatially resolved NIRS technology and has a comparable source-detector separation, so it might to some extent be an issue of the specific NIRS instrument. ${ }^{20,21}$ In a study on newborn lambs subjected to hypoxia, Wong et al. ${ }^{11}$, also using the NIRO-300 instrument and assuming a fixed AVratio of 0.25 , found that cerebral NIRS reflected $51 \%$ of the changes in weighted arterial and venous cerebral oxygenation. This study indicates that NIRS is sensitive to changes in AVratio. Wong et al. used the NIRO-300 device and measured reference co-oximetry in the extremes (range 13.7 to 74.4 ) as in our study. Anatomical differences between species may only partly explain the different accuracy with the NIRS-probe on lambs at 3 to 6 days possibly having a better fit than on piglets.

The most significant difference between the study from Wong et al. and our study may be that we used hypotension to reduce cerebral oxygenation. We minimized the potential vasoactive effects of arterial $\mathrm{O}_{2}$ and $\mathrm{CO}_{2}$ on arterioles by keeping $\mathrm{S}_{\mathrm{a}} \mathrm{O}_{2}$ and $\mathrm{P}_{\mathrm{a}} \mathrm{CO}_{2}$ stable during the phases with manipulation 
of MABP, and thus the physiological effects in play are most likely related to arterial and venous pressures. The vena cava balloon was inflated until MABP was reduced to the level required. The most likely mode of action of this intervention is through decreased venous return from the lower body leading to reduced preload of the heart. However, we did not measure the central venous pressure to confirm this or quantify the effect. We speculate that the reduced preload lowered the venous volumes of the upper limbs and the head, while cerebral arteries actively dilate as a response to the arterial hypotension due to the myogenic response. ${ }^{10,18}$ Thus, arterial blood volume dominates the $\mathrm{CBV}$ at low MABP. This may explain the poor response of NIRS in terms of cerebral volume-weighted oxygenation when assuming a fixed AV-ratio, because NIRS increasingly reflects arterial blood, leading to higher results compared to fixed AV-ratio, as blood pressure is reduced. The accuracy of NIRS-oximeters is not possible to determine in vivo, due to the uncertainty of the AV-ratio. Therefore, the in vitro accuracy is important. In the original paper describing the method of spatially resolved spectroscopy, very good accuracy in a blood-lipid phantom was demonstrated over oxygenation ranging from less than $20 \%$ to nearly $100 \%{ }^{22}$ In a more recent study using the same phantom, however, different NIRS-devices differed significantly as regard to their response to deoxygenation, e.g., the NIRO300 change was only 53\% of the INVOS5100 change, ${ }^{20}$ whereas the INVOS5100 change came close to that of a reference standard. ${ }^{23}$ Another study reported a relative sensitivity to deoxygenation induced by a range of stimuli in young swine of $\sim 50 \%$ using NIRO-300 compared to the INVOS. ${ }^{21}$ Thus, if we assume that the NIRO300 underestimates true deoxygenation by $50 \%$, then the underestimation found in the present data must include yet another factor. We suggest that this is an increase in the AV-ratio.

CBV in newborn piglets has previously been found to $\sim 4.5 \mathrm{ml} / 100 \mathrm{~g}$ measured by radiolabeled erythrocytes. ${ }^{24}$ While we did not attempt to measure CBV in absolute terms, we were able to calculate changes over time from the trends of oxy- and deoxyhemoglobin concentrations that are provided by the NIRO device in $\mu \mathrm{mol} / \mathrm{ml}$ (brain tissue). These changes were small, but in the expected direction during physiological challenges. For example, lowering MABP by $40 \mathrm{mmHg}$ resulted in a reduction of $\sim 0.44 \mathrm{ml} / 100 \mathrm{~g}$ or $10 \%$ of total CBV. A lowering of MABP by $40 \mathrm{mmHg}$ equals an increase of 0.28 in the AV-ratio. Previously, Lee et al. ${ }^{25}$ reported a comparable increase in the arterial volume fraction from 0.25 to 0.40 in response to hypercapnia, as the volume increase in the venous compartment was only approximately half of that in the arterial compartment. The lower threshold of the autoregulatory compensative capacity has been reported as an MABP of $30 \mathrm{mmHg}$ or less in newborn children ${ }^{26}$ and 29 to $48 \mathrm{mmHg}$ in newborn piglets, ${ }^{27,28}$ although wide interindividual variations are likely to exist. ${ }^{18}$ MABP below the lower threshold of autoregulation would result in a pressure-passive reduction of cerebral blood flow. Our results suggest that cerebral autoregulation is able to buffer the loss of CBV until the lower threshold of the autoregulation is reached. This could explain why the reduction in CBV was small compared to the increase of the AV-ratio. The variability of the data, most likely due to the low precision of cerebral oximetry by NIRS, made it impossible to examine any nonlinear relation between $\mathrm{CBV}$ and $\mathrm{AV}$-ratio changes, which could have illustrated a possible change in vascular behavior below the autoregulatory threshold.

\subsection{Implications}

Validation of cerebral NIRS against blood oxygenation in adults and infants has predominantly focused on situations of hypoxia and low- or high-blood $\mathrm{CO}_{2}$ content (hypo- or hypercapnia). ${ }^{11,19}$ However, as hypotension is a condition commonly treated in sick infants, ${ }^{29}$ validation under conditions resembling hypotension are also highly relevant. In healthy adult volunteers, cerebral oxygenation measured by NIRS during hypovolemia has been studied experimentally by applying lower body negative pressure. ${ }^{30,31}$ Literature on the effects of hypotension on NIRS oximetry measures in neonatal populations is limited. In preterm infants, mild short-term hypotensive episodes did not lower the cerebral oxygenation estimated by NIRS. ${ }^{32}$ Our study indicates that changes in the AV-ratio during hypotension should be taken into account and that we might underestimate the true reduction in tissue oxygenation by NIRS in this situation, as NIRS increasingly reflects arterial blood. Ideally, validation between NIRS oximetry and cerebral blood oxygenation should be adjusted by simultaneous and independent estimations of changes in the AV-ratio. This is not currently possible, therefore, the alternative is to investigate changes in the AV-ratio during pathophysiological conditions that are relevant in sick infants and take this knowledge into account when using cerebral oximetry in clinical practice.

\subsection{Strengths and Limitations of the Study}

Our piglet model allowed blood sampling from the superior sagittal sinus, draining the cerebral cortex, and thus minimizing the risk of contamination from the extracerebral veins. ${ }^{33}$ Once placed, the NIRS probe was secured by nontransparent bandage and not repositioned. This prevented reposition noise, where optic heterogeneity in the tissue as well as heterogeneity of blood flow in the brain would add within-subject variation. We were able to decrease MABP to very low levels, allowing an examination of NIRS in extreme situations of potential clinical relevance. In Eq. (1), we used human DPF due to lack of piglet data. DPF is a measure of the time it takes the light to travel through the tissue relative to what would be expected from a direct flight from source to detector. It depends on the optical characteristics of the tissue. We do know that the piglet brain is more mature at the time of birth with more developed myelination. This will increase DPF. Using too low DPF will not influence the estimate of rStO2, but will lead to an underestimation of the absolute changes in CBV.

Approximately $1 / 3$ of the calculated AV-ratios were above 1 or below 0 . The data were retained in our analysis to avoid statistical bias. In our calculation, the $\mathrm{AV}$-ratio is determined by $\mathrm{rStO}_{2, \mathrm{NIRS}}, \mathrm{S}_{\mathrm{a}} \mathrm{O}_{2}$, and $\mathrm{S}_{\mathrm{v}} \mathrm{O}_{2}$ [Eq. (3)]. The $\mathrm{rStO}_{2}$ estimate of NIRO-300 has been shown to estimate lower baseline values compared to other devices in neonates ${ }^{34}$ and on the adult forearm. ${ }^{35}$ Also, a study in a blood-lipid phantom revealed significant difference in $\mathrm{rStO}_{2}$ estimates between three commercial devices, including the NIRO-300. ${ }^{20,21}$ Errors in the measurement of $\mathrm{S}_{\mathrm{v}} \mathrm{O}_{2}$ would equally be able to estimate AV-ratios below 0 if the venous samples were not taken strictly anaerobically. Air in the syringe was carefully avoided and, if present, quickly removed when blood samples were drawn and the blood samples were analyzed immediately after sampling. The procedure did not differ from that in clinical practice. Although air-contamination cannot be ruled out in some samples, it is unlikely to be the sole cause of the large number of data points outside 
the physiological range. Wong et al. ${ }^{11}$ excluded eight nonphysiological values out of 49 during the analysis of the AVratio and $\triangle \mathrm{CBV}$. Finally, imprecision of NIRS oximetry may explain some of the nonphysiological data. It has been well-documented that irrespective of the NIRS instrument the precision of the $\mathrm{rStO}_{2}$ estimate is about $5 \%$ to $6 \% .^{36,37}$ This means that a single $\mathrm{rStO}_{2}$ estimate could be $\pm 15 \%$ from the "true" oxygenation, thus NIRS values below $\mathrm{SvO}_{2}$ could simply be due to random noise in the NIRS signal. Another limitation of our study is the fact that it was performed in newborn piglets. There are obvious differences between piglets and neonates. Newborn piglet brains average weight is $30 \mathrm{~g}$, whereas extremely preterm infant brains weigh at least $100 \mathrm{~g}$. The NIRS sensor was designed for human use and a source-detector distance of $4 \mathrm{~cm}$ might probe beyond the brain when used in a piglet including extracerebral tissue, such as tongue and basis cranii. Finally, the study was part of a larger setup where the effect of dopamine-infusion on cerebral autoregulation was studied. The piglets received dopamine when half of the blood samples were drawn. Dopamine is an inotrope affecting vascular resistance and cardiac output through dopaminergic, $\alpha_{1}$ - and $\beta_{2}$-adrenoceptors in arteries and the heart. ${ }^{38}$ Thus, dopamine has the potential to change cerebral blood flow. Importantly, dopamine did not affect the relationship between $\mathrm{rStO}_{2, \mathrm{NIRS}}$ and $\mathrm{rStO}_{2, \mathrm{COX}}$, between $\triangle \mathrm{CBV}$ and MABP, nor between the AV-ratio and MABP. Dopamine did not affect autoregulation (manuscript submitted). We are, therefore, confident that our results are relevant in a wider context.

\section{Conclusion}

NIRS oximetry had a poor response to cerebral hypoxia during hypotension when compared to a reference co-oximetry value assuming a fixed AV-ratio. This may partly be explained by the inaccuracy of the NIRS-device, but our data indicate that the AV-ratio changes during hypotension and affects NIRS estimates of cerebral oxygenation. Further research in NIRS oximetry in pathophysiological states is needed to help interpret future results for clinical use.

\section{Disclosures}

No conflicts of interest to declare.

\section{References}

1. I. Seri, "Management of hypotension and low systemic blood flow in the very low birth weight neonate during the first postnatal week," J. Perinatol. 26(Suppl 1), S8-S13 (2006).

2. S. Noori, T. A. Stavroudis, and I. Seri, "Systemic and cerebral hemodynamics during the transitional period after premature birth," Clin. Perinatol. 36(4), 723-736 (2009).

3. B. V. Bhat and N. Plakkal, "Management of shock in neonates," Indian J. Pediatr. 82(10), 923-929 (2015).

4. E. M. Dempsey and K. J. Barrington, "Treating hypotension in the preterm infant: when and with what: a critical and systematic review," J. Perinatol. 27(8), 469-478 (2007).

5. S. Noori and I. Seri, "Evidence-based versus pathophysiology-based approach to diagnosis and treatment of neonatal cardiovascular compromise," Semin. Fetal Neonat. Med. 20(4), 238-245 (2015).

6 . V. Pollard et al., "Validation in volunteers of a near-infrared spectroscope for monitoring brain oxygenation in vivo," Anesth. Analg. 82, 269-277 (1996).

7. S. Hyttel-Sorensen et al., "Cerebral near infrared spectroscopy oximetry in extremely preterm infants: phase II randomised clinical trial," Br. Med. J. 350, g7635-g7635 (2015).
8. A. Pellicer and M. D. C. Bravo, "Near-infrared spectroscopy: a methodology-focused review," Semin. Fetal Neonat. Med. 16(1), 42-49 (2011).

9. H. An and W. Lin, "Cerebral venous and arterial blood volumes can be estimated separately in humans using magnetic resonance imaging," Magn. Reson. Med. 48(4), 583-588 (2002).

10. G. Greisen, "Autoregulation of cerebral blood flow in newborn babies," Early Hum. Dev. 81(5), 423-428 (2005).

11. F. Y. Wong et al., "Cerebral arterial and venous contributions to tissue oxygenation index measured using spatially resolved spectroscopy in newborn lambs," J. Am. Soc. Anesthesiol. 113(6), 13851391 (2010).

12. Y. A. Wickramasinghe et al., "Plethysmographic validation of near infrared spectroscopic monitoring of cerebral blood volume," Arch. Dis. Child. 67, 407-411 (1992).

13. A. Duncan et al., "Optical pathlength measurements on adult head, calf and forearm and the head of the newborn infant using phase resolved optical spectroscopy," Phys. Med. Biol. 40(2), 295-304 (1995).

14. C. Elwell, A Practical User's Guide to Near Infrared Spectroscopy, Hamamatsu Photonics KK, Hamamatsu City, Japan (1995).

15. A. A. Lammertsma et al., "In vivo measurement of regional cerebral haematocrit using positron emission tomography," J. Cereb. Blood Flow Metab. 4(3), 317-322 (1984).

16. S. R. Nelson, M. L. Mantz, and J. A. Maxwell, "Use of specific gravity in the measurement of cerebral edema," J. Appl. Physiol. 30(2), 268271 (1971).

17. L. C. Henson et al., "Accuracy of a cerebral oximeter in healthy volunteers under conditions of isocapnic hypoxia," Anesthesiology 88, 58-65 (1998).

18. N. Brew, D. Walker, and F. Y. Wong, "Cerebral vascular regulation and brain injury in preterm infants," Am. J. Physiol. Regul. Integr. Comp. Physiol. 306(11), R773-R786 (2014).

19. M. H. Watzman et al., "Arterial and venous contributions to near-infrared cerebal oxumetry," Anesthesiology 93(4), 947-953 (2000).

20. S. Hyttel-Sorensen et al., "Calibration of a prototype NIRS oximeter against two commercial devices on a blood-lipid phantom," Biomed. Opt. Express 4(9), 1662-1672 (2013).

21. R. E. Gagnon et al., "Comparison of two spatially resolved NIRS oxygenation indices," J. Clin. Monit. Comput. 17(7-8), 385-391 (2002).

22. S. Suzuki et al., "A tissue oxygenation monitor using NIR spatially resolved spectroscopym," Proc. SPIE 3597, 582 (1999).

23. S. Kleiser et al., "Comparison of tissue oximeters on a liquid phantom with adjustable optical properties," Biomed. Opt. Express 7(8), 2973 (2016).

24. N. C. Brun et al., "Near-infrared monitoring of cerebral tissue oxygen saturation and blood volume in newborn piglets," Am. J. Physiol. 273(2 Pt 2), H682-H686 (1997).

25. S. P. Lee et al., "Relative changes of cerebral arterial and venous blood volumes during increased cerebral blood flow: implications for bold fMRI," Magn. Reson. Med. 45(5), 791-800 (2001).

26. L. Tyszczuk et al., "Cerebral blood flow is independent of mean arterial blood pressure in preterm infants undergoing intensive care," Pediatrics 102(2), 337-341 (1998).

27. K. M. Brady et al., "Noninvasive autoregulation monitoring with and without intracranial pressure in the naïve piglet brain," Anesth. Analg. 111(1), 191-195 (2010).

28. J. K. Lee et al., "Cerebrovascular reactivity measured by near-infrared spectroscopy," Stroke 40(5), 1820-1826 (2009).

29. K. J. Barrington, "Hypotension and shock in the preterm infant," Semin. Fetal Neonat. Med. 13(1), 16-23 (2008).

30. V. L. Kay and C. A. Rickards, "The role of cerebral oxygenation and regional cerebral blood flow on tolerance to central hypovolemia," Am. J. Physiol. Regul. Integr. Comp. Physiol. 310(4), R375-R383 (2016).

31. C. A. Rickards et al., "Coupling between arterial pressure, cerebral blood velocity, and cerebral tissue oxygenation with spontaneous and forced oscillations," Physiol. Meas. 36(4), 785-801 (2015).

32. C. Binder-Heschl et al., "Borderline-hypotension, how does it influence the cerebral tissue oxygenation?," J. Paediatr. Child Health 51(July), 45 (2015).

33. B. Schaller, "Physiology of cerebral venous blood flow: from experimental data in animals to normal function in humans," Brain Res. Rev. 46(3), 243-260 (2004). 
34. M. Pocivalnik et al., "Regional tissue oxygen saturation: comparability and reproducibility of different devices," J. Biomed. Opt. 16(5), 057004 (2011).

35. S. Hyttel-Sorensen et al., "Tissue oximetry: a comparison of mean values of regional tissue saturation, reproducibility and dynamic range of four NIRS-instruments on the human forearm," Biomed. Opt. Express 2(11), 3047 (2011).

36. T. W. Hessel, S. Hyttel-Sorensen, and G. Greisen, "Cerebral oxygenation after birth-a comparison of INVOS ${ }^{\circledR}$ and FORE-SIGHTTM NIRS oximeters," Acta Paediatr. 103, 488-493 (2014).
37. S. Hyttel-Sorensen, T. W. Hessel, and G. Greisen, "Peripheral tissue oximetry: comparing three commercial near-infrared spectroscopy oximeters on the forearm," J. Clin. Monit. Comput. 28(2), 149-155 (2014).

38. S. Noori and I. Seri, "Neonatal blood pressure support: the use of inotropes, lusitropes, and other vasopressor agents," Clin. Perinatol. 39(1), 221-238 (2012).

Biographies for the authors are not available. 